\title{
Gaussian Samples, Regular Simplices, and Exchangeability*
}

\author{
Y. M. Baryshnikov \\ School of Mathematics, University of Hull, \\ Hull, Humberside HU6 7RX, England \\ y.baryshnikov@maths.hull.ac.uk
}

Abstract. The interchangeable Goodman-Pollack-type random finite sets are characterized.

\section{Introduction}

Choose a finite subset $\mathbf{P}$ in Euclidean space $V=\mathbf{R}^{N}$, subject it to a random rotation $\mathbf{r}$ and project the resulting set $\mathbf{r P}$ in a fixed way onto a linear space (of smaller dimension) $\pi: V \rightarrow W=\mathbf{R}^{n}$. The random set $\pi \mathbf{r P}$ thus obtained is a natural alternative to the standard paradigm of geometric probabilities, in which random finite sets are usually assumed to be iid samples of points chosen independently accordingly to some (the same for all points) probability distribution.

This mechanism to generate random finite subsets was suggested by J. E. Goodman and R. Pollack and pursued further by Affentranger and Schneider [1]. They take as the initial finite set $P$ the vertices of a regular simplex in $V$ and assume the random rotation to be distributed according to the natural (shift-invariant or Haar) probability measure on the orthogonal group. Investigating this case they observed an amazing coincidence: the convex hull of the projected set has the same expected number of facets (i.e., $(n-1)$ dimensional faces) as the convex hull of the standard Gaussian iid sample in $W=\mathbf{R}^{n}$ (calculated in [5]).

This phenomenon was explained by Vitale and the author in [3]. We have shown that if the randomly projected vertices of the simplex are further subjected to a certain random affine transformation which is independent of the random rotation preceding the projection, then the resulting point set has the same distribution as the standard Gaussian iid sample. Of course, as the number of faces (of any dimension) is an affine invariant

* This work was done during the author's stay at the Fachbereich Mathematik, Universität Osnabrück, and was supported by the Deutsche Forschungsgemeinschaft. 
of the convex hull, the distributions of this number under the standard Gaussian model and under the Goodman-Pollack model coincide, which explains the observation of Affentranger and Schneider.

This result leads to the following natural questions: Is the Gaussian distribution so special? That is, could it happen that choosing some other set of points instead of the vertices of regular simplex one obtains (by randomly rotating and projecting) the random set which is affinely equivalent to the iid sample from a non-Gaussian distribution? What if one takes into account not the affine similarity of the random point sets but just their combinatorial structure? What if one drops the independence condition?

To formulate these questions more precisely, we fix the notation. Assume that the set $\mathbf{P}$ contains $m>n+1$ points $\mathbf{P}=\left\{p_{1}, \ldots, p_{m}\right\}$, so that its generic projection results in a sample of size $m$ in $W$. Without losing generality we can assume that the affine span of $\mathbf{P}$ is the whole $V$ (and, hence, $m>N$ ). Otherwise, the restriction of the random projection to this spanning affine subspace gives the Goodman-Pollack model again. The configuration space of $m$-tuples of points in $W$ we denote as $\operatorname{Conf}_{m}(W)$. Apparently, this configuration space is just the $m$-fold product of $W$ by itself.

The group $A f f(W)$ of affine invertible transformations of $W$ acts on $\operatorname{Conf}_{m}(W)$ (by the diagonal action). The factorspace of $\operatorname{Conf}_{m}(W)$ by the action of $A f f(W)$ is denoted by $A f f_{m}(W)=\operatorname{Conf}_{m}(W) / A f f$. An element of the space encodes the shape of the configuration modulo affine equivalence. This topological space is not a manifold (it is not even Hausdorff). However, if we consider an open dense subset $\operatorname{Conf}_{m}^{0}(W) \subset \operatorname{Conf}_{m}(W)$ consisting of $m$-tuples of points whose first $n+1$ points are in general position, that is, such that their affine span is the whole of $W$, then the factor of $\operatorname{Conf}_{m}^{0}(W)$ by $A f f(W)$ is diffeomorphic to the $(m-n-1)$-fold product of $W$ with itself. Indeed, these first $(n+1)$ points forming a nondegenerate simplex in $W$ can be sent to the origin and $n$ prespecified basis vectors, respectively, by a uniquely defined affine transformation, after which the remaining $m-n-1$ points can be chosen arbitrarily. The space $\operatorname{Conf}_{m}^{0}(W) / A f f(W)=$ $W^{m-n-1}$ is denoted as $\operatorname{Aff}_{m}^{0}(W)$.

A further loss of information is to drop any geometry altogether and to characterize any configuration by its combinatorial type, that is, by specifying the signs of oriented volumes of simplices spanned by $(n+1)$-tuples of points of the considered $m$-configuration in $\operatorname{Conf}_{m}(W)$. The combinatorial type fixes such discrete invariants as the face lattice of the convex hull of the point set or of any of its subsets, the oriented matroid corresponding to the configuration, etc. The combinatorial types of $m$ tuples in $W$ form a finite set $\operatorname{Comb}_{m}(W)$. There exists the sequence of mappings loosening the structure:

$$
\operatorname{Conf}_{m}(W) \stackrel{\text { Aff }}{\rightarrow} \operatorname{Aff}_{m}(W) \stackrel{s g n}{\rightarrow} \operatorname{Comb}_{m}(W),
$$

where /Aff is the factoring by the affine group, and $s g n$ is the passing to the combinatorial type.

Any subset $\mathbf{P} \subset V$ consisting of $m$ points defines a probability distribution on Conf $_{m}(W)$ by the Goodman-Pollack mechanism: one applies a random rotation $\mathbf{r}$ (distributed uniformly, that is, with the Haar measure on $S O(V)$ ) to $\mathbf{P}$ and projects the resulting set $\mathbf{r P}$ to $V$. The distribution on $\operatorname{Conf}_{m}(W)$ thus obtained we denote as $G P(W)$. The points of $\mathbf{P}$ generate affinely the whole of $V$, so that there exists among these points an $(N+1)$-tuple in general position, and, consequently, an $(n+1)$-tuple in general 
position. We can assume, without loss of generality, that the points of the tuple are $n+1$ first points in $\mathbf{P}$. Apparently, for almost all $\mathbf{r} \in S O(V)$ the projection of $\mathbf{r P}$ into $W$ is a configuration in $\operatorname{Conf}_{m}^{0}(W)$, so that the $G P(\mathbf{P})$-content of $\mathbf{A f f}_{m}^{0}(W) \subset \mathbf{A f f}_{m}(W)$ is 1 .

The distribution $G$ of $m$-tuples of iid points from $W$ with Gaussian density $(2 \pi)^{-n / 2} e^{-x^{2} / 2}$ is Gaussian itself and is denoted as $G^{m}$. Once again, it is obvious that the $G^{m}$-content of $\operatorname{Conf}_{m}^{0}(W)$ is 1 .

Measures $G P(\mathbf{P})$ and $G^{m}$ supported by $\operatorname{Conf}_{m}^{0}(W)$ can be mapped to certain measures on $\mathbf{A f f}_{m}^{0}(W)$ and to $\operatorname{Comb}_{m}(W)$, in which case we add subscripts Aff and Comb, respectively.

In these terms the result of Schneider and Affentranger is that the projections of the distributions $G P(\mathbf{P})$ and $G^{m}$ into $\operatorname{Comb}_{m}(W)$ coincide, $G P(\mathbf{P})_{\text {Comb }}=G_{\mathbf{C o m b}}^{m}$ (or, rather, that expectations of certain functions of the combinatorial type with respect to these two distributions coincide) when $\mathbf{P}$ is a regular simplex in $V$. The explanation given in [3] for this phenomenon establishes that the projections of these distributions to $\mathbf{A f f}_{m}^{0}(W)$ already coincide: $G P(\mathbf{P})_{\mathrm{Aff}}=G_{\mathrm{Aff}}^{m}$.

Hence, the questions posed above can now be formulated as follows: Is it possible, by varying the point set $\mathbf{P}$, to achieve the distribution of the affine types in the GoodmanPollack model (that is, the distributions $G P(\mathbf{P})_{\text {Aff }}$ into $\mathbf{A f f}_{m}(W)$ ) coinciding with the distribution of affine types of the iid sample from a certain distribution $P$, other than Gaussian, or at least the same question for the distribution of combinatorial types?

It turns out that all the questions are answered in the negative and that the Gaussian model is rigid, even if one drops the independence assumption insofar as the interchangeability of the points in the random set is maintained. That is what the main result of this note says:

Theorem 1. Let $\mathbf{P} \subset V$ be a set of $m>n+1$ points, $n=\operatorname{dim} W ;$ let $G P(\mathbf{P})$ be the corresponding Goodman-Pollack-type distribution of $m$-configurations in $W$; and let $G P_{\mathbf{C o m b}}(\mathbf{P})$ be the corresponding distribution of the combinatorial types of $m$-point configurations in $W$. Then if $G P(\mathbf{P})_{\text {Comb }}$ is symmetric (or interchangeable), that is, if the permutations of points do not affect the distribution, then $\mathbf{P}$ is the vertex set of a regular simplex in $V$.

\section{Proof of Theorem 1}

Choose a subset $\mathbf{P}^{\prime}$ of $\mathbf{P}$ consisting of $n+2$ points and denote by $L$ its affine span. Let $K=\operatorname{Ker} \pi$ be the kernel of the projection in the Goodman-Pollack model; $\operatorname{dim} K=$ $\operatorname{dim} V-\operatorname{dim} W=N-n$. Apparently, the combinatorial type of the projection of $\mathbf{r P}^{\prime}$ is that of the projection of $\mathbf{P}^{\prime}$ along $L \cap \mathbf{r}^{-1} K$.

Lemma 1. The dimension of $L$ is $(n+1)$ and therefore $\mathbf{P}^{\prime}$ is the set of vertices of a nondegenerate simplex in $L$.

Proof. If the dimension of $L$ is less than $n+1$, then for almost all rotations $\mathbf{r}$ the intersection $L \cap \mathbf{r}^{-1} K$ is a point and therefore the combinatorial type of $\pi \mathbf{r P}^{\prime}$ is constant almost surely. The symmetry with respect to permutations of indices implies that for 
each pair of points in $\mathbf{P}^{\prime}$ the segment joining them is an edge of the convex hull of $\mathbf{P}^{\prime}$ (otherwise all these segments were not edges). It is possible only if the points from $\mathbf{P}^{\prime}$ are vertices of a nondegenerate simplex.

Choose some barycentric coordinates $x_{i}, i=1, \ldots, n+2, \sum x_{i}=0$, in $L$ such that the simplex spanned by $\mathbf{P}^{\prime}$ is given by the inequalities $x_{i} \geq c_{i}, c_{i} \in \mathbf{R}, i=1, \ldots, n+2$. We provide the linear space $L$ with Euclidean metric by the restriction of the Euclidean metric from the ambient space $V$.

The intersection $L_{\mathbf{r}}=L \cap \mathbf{r}^{-1} K$ is one-dimensional almost surely (with respect to Haar measure on $\mathbf{r}$ 's). Fixing orientations of $V, L$, and $K$, we obtain an orientation on the line $L_{\mathbf{r}}$. The displacement of this oriented line to the origin of $K$ we denote by $L_{\mathbf{r}}^{0}$, and the "positive" ray on $L_{\mathrm{r}}^{0}$ by $R_{\mathrm{r}}$.

Lemma 2. The distribution of the intersection of $R_{\mathrm{r}}$ with the unit sphere in $L$ is a uniformly distributed random point (that is, its distribution is rotationally invariant).

Proof. The invariance of the distribution of $\mathbf{r}^{-1} K$ with respect to actions of elements of $S O(V)$ implies the invariance of the distribution of $L_{\mathbf{r}}^{0}$ with respect to the action of the subgroup $S O(L)$.

Hence, the combinatorial type of the random projection of $\mathbf{P}^{\prime}$ coincides 'with the combinatorial type of the projection of $\mathbf{P}^{\prime}$ along $L_{\mathbf{r}}$, and, by the Lemma 2 , with the combinatorial type of the projection of the simplex $\mathbf{P}^{\prime}$ along the line $L_{\mathbf{r}}^{0}$ equidistributed with the line passing through the origin and the point chosen at random from the unit sphere. The probability that the $(n-1)$-simplex $\Delta$ spanned by points $\mathbf{P}^{\prime}-\{i, j\}$ goes to a facet of the convex hull of the projection of $\mathbf{P}^{\prime}$ along $L_{\mathbf{r}}$ is the spherical content of the wedge defined by inequalities $x_{i} \geq 0, x_{j} \leq 0$. Indeed, let $L_{\mathrm{r}}^{\prime}$ be a line parallel to $L_{\mathbf{r}}$ and passing through an interior point $x$ of the simplex $\Delta$. Then the image of the simplex is a facet if and only if the affine hyperplane in $L$ spanned by the simplex and $L_{\mathrm{r}}^{\prime}$ does not intersect the interior of the simplex spanned by $\mathbf{P}^{\prime}$, or, equivalently, if the line $L_{\mathbf{r}}^{0}$ does not intersect the interior of the tangent cone to $\mathbf{P}^{\prime}$ along $\Delta$. This latter is exactly the wedge $\left\{x_{i} \geq 0, x_{j} \leq 0\right\}$.

The spherical content of the wedge is equal to the spherical distance between the normed covectors $x_{i} /\left|x_{i}\right|$ and $x_{j} /\left|x_{j}\right|$. The symmetry implies that all the contents and hence all the distances are equal for all pairs $i, j \in \mathbf{P}^{\prime}$. This immediately implies that the simplex defined by inequalities $x_{i} \geq c_{i}, c_{i} \in \mathbf{R}, i=1, \ldots, n+2$, is regular in $L$ and has equal edges. As the reasoning is valid for any subset $\mathbf{P}^{\prime} \subset \mathbf{P}$, all pairwise distances between points of $\mathbf{P}$ are equal and $\mathbf{P}$ is the set of vertices of a regular simplex in $V$.

\section{Concluding Remarks}

A. Theorem 1 implies that the resulting distribution in the combinatorially interchangeable Goodman-Pollack model is affinely equivalent to the Gaussian distribution in the standard model. This (annoying) inevitability of the Gaussian distribution makes one recollect the variety of the "de Finetti" style results, especially the Theorem of Shoenbergtype [2], [4], but as of now, we do not see any direct connection. 
B. The proof of the theorem can be shortened a bit, but in its present form it proves somewhat more: we do not need, in fact, the invariance of the distribution of the combinatorial types with respect to the whole permutation group. The 2-transitivity of the symmetry group is enough. Just the transitivity would not suffice as the simple example of the random projection of a regular cube to the plane shows. The condition that $m>n+1$ also cannot be improved: all plane triangles are affinely equivalent and do not carry any information about the original distribution on $\operatorname{Conf}(W)$.

C. An expansion of the results can be done for other than symmetric (or 2-transitive) symmetry groups if one considers zonotopes. A zonotope is the Minkowsky sum of segments or, equivalently, the convex hull of image of the vertices of the unit cube under some affine mapping. Analogously to the general story about convex polytopes, one can consider the standard random zonotopes, whose generating segments are chosen independently from some distribution (if one is after the shape of the zonotope, one can assume that the segments all have one end at the origin and identify random segments with random vectors), and the G-P or projection type zonotopes, that is, the projections along a subspace of a randomly rotated affine cube.

There is a natural group $G$ acting on the $2^{N}$ vertices of the unit cube: the group of its symmetries. It is generated by the transpositions of the coordinates and by the reflections in the hyperplanes $\left\{x_{t}=\frac{1}{2}\right\}$. This group is, clearly, much smaller than the group of permutations of the vertices of the cube and is not even 2-transitive. However, it is large enough to prove an analogue of Theorem 1. We preserve the notation of Section 1. Let $\mathbf{C}$ be the set of vertices of a parallelepiped in $V=\mathbf{R}^{N}$, so that $m=\# \mathbf{C}=2^{N}$, and let $G P_{\mathbf{C o m b}}(\mathbf{C})$ be the distribution of the combinatorial types of the composition of random rotation and projection of $\mathbf{C}$.

Theorem 2. If $G P_{\mathrm{Comb}}(\mathrm{C})$ is invariant with respect to the natural action of the group of symmetries of cube $G$, then the convex hull of $\mathbf{C}$ is a regular cube, and the distribution of the affine type of the Goodman-Pollack sample coincides with that of the Standard Gaussian model, that is, the model where all the segments are independent Gaussian vectors.

The proof is analogous to the proof of Theorem 1.

\section{References}

1. F. Affentranger and R. Schneider, Random projections of regular simplices. Discrete Comput. Geom. 7 (1992), 219-226.

2. D. J. Aldous, Exchangeability and Related Topics, Lecture Notes in Mathematics, Vol. 1117, SpringerVerlag, New York, 1985.

3. Yu. Baryshnikov and R. Vitale, Regular simplices and Gaussian samples, Discrete Comput. Geom. 11 (1994), 141-147.

4. P. Diaconis and D. Freedman, A dozen de Finetti-style results in search of a theory, Ann. Inst. H. Poincaré 23 (1987), 397-423.

5. H. Raynaud, Sur l'enveloppe convexe des nuages de points aléatoires dans $\mathrm{R}^{n}$, J. Appl. Probab. 7 (1970), 35-48.

Received June 14, 1995, and in revised form October 16, 1995. 